\title{
Gastric Schwannoma: A Case Report and Review of Literature
}

\author{
M. R. Sreevathsa • Gotam Pipara
}

Received: 8 June 2014 / Accepted: 11 December 2014 /Published online: 18 March 2015

(C) Indian Association of Surgical Oncology 2015

\begin{abstract}
Schwannomas are usually benign, slow growing tumors, that originate from any nerve that has a Schwann cell sheath. Here, we report the case of a 40 year-old female patient with an incidentally noted submucosal gastric tumor while being evaluated for cervical lymphadenopathy as a part of workup for lymphoma. She underwent sleeve resection of the stomach under suspicion of a gastrointestinal stromal tumor, but postoperative histopathological and immunohistochemical findings confirmed the diagnosis of shwannoma. Although schwannomas are mostly benign, they are often indistinguishable preoperatively from malignant tumors such as gastrointestinal stromal tumors. Therefore, resection is the treatment of choice for all such tumors.
\end{abstract}

Keywords Gastric schwannoma · GIST · Immunohistochemistry $\cdot$ Sleeve resection

\section{Case Report}

A 48 year old lady with cervical lymphadenopathy was subjected to ultrasound of the abdomen for ruling out splenomegaly as a part of work up for lymphoma. Ultrasound of the abdomen (Fig. 1) revealed a well defined lobulated

M. R. Sreevathsa $(\bowtie)$

Department of General Surgery, M.S. Ramaiah Medical College and

Hospitals, M.S.R.I.T Post, M.S.R. Nagar,

Bangalore 560054, Karnataka, India

e-mail: chee_vaccha@yahoo.co.in

G. Pipara

M.S. Ramaiah Medical College and Hospitals,

Bangalore 560054, India

e-mail: gotampipara@yahoo.com hypoechoic mass lesion in the epigastric region located between the left lobe of liver and gastric wall. It was $4.2 \times$ $4.1 \mathrm{~cm}$ in size. Computed tomography scan showed well defined uniformly enhancing gastric fundal wall mass, (Fig. 2) most likely to be GIST (gastrointestinal stromal tumour) with celiac axis and periportal nodal enlargement. The mass was partly exophytic and partly projecting into the lumen causing smooth indentation. The patient then underwent upper GI endoscopy which showed a sub mucosal bulge on the anterior wall of the fundus of the stomach. An endosonography was done using a linear scope which showed a large submucosal mixed echoic mass with few areas of necrosis in body of stomach mainly on greater curvature measurng $5 \times 4 \mathrm{~cm}$ arising from muscularis propria. Two celiac nodes, hypoechoic round with uniform echotexture were also seen. FDG pet scan showed metabolically active tumor mostly representing GIST (Gastrointestinal stromal tumour) from body of the stomach (Fig. 3).

A provisional diagnosis of submucosal mass, which could be most likely GIST, was made and fine needle aspiration cytology was done from both the lesion and the nodes with endosonoguidance. The FNA from gastric submucosal lesion showed features suggestive of spindle cell tumor while the celiac nodes showed reactive hyperplasia. A preoperative diagnosis of GIST arising from anterior wall of proximal stomach was made. The patient was subjected to laparoscopic examination which revealed an exophytic solid tumor measuring $6 \times 7 \mathrm{~cm}$ arising from the anterior wall of the junction of body and fundus. The medial margin was at the lesser curvature and lateral margin was just short of greater curvature. In order to get a good tumor negative margin laparoscopy was converted to minilaparotomy and sleeve resection of the tumor was done using linear staplers. Histopathology revealed a submucosal tumor with features suggestive of spindle cell tumor (Fig. 4). IHC done showed positivity 


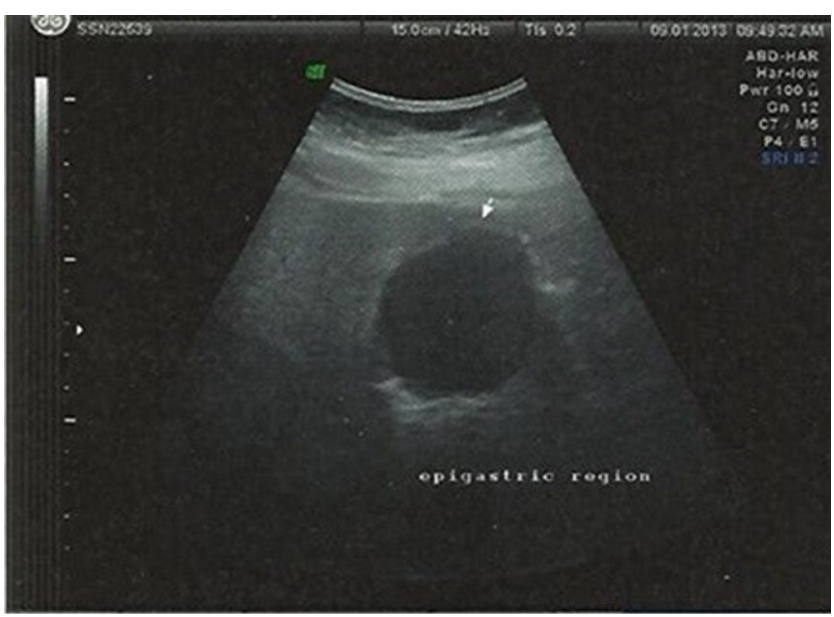

Fig. 1 Ultrasonography image

for s100 and negative for actin, CD34, CD117 and desmin which confirmed a diagnosis of schwannoma. Patient had an uneventful recovery and cervical LN biopsy confirmed tuberculosis.

\section{Discussion}

Gastrointestinal mesenchymal tumors are a group of tumors originating from the mesenchymal stem cells of the gastrointestinal tract. They consist of gastrointestinal stromal tumor (GIST), leiomyoma or leiomyosarcoma, and schwannoma [1, 2]. Gastric schwannoma contributes to $0.2 \%$ of all gastric tumors overall, $6.3 \%$ of gastric mesenchymal tumours and $4 \%$ of all benign tumors of the stomach [3]. Malignant schwannoma are very rare, as only 8 cases have been reported in the literature till date $[4,5]$. These are encapsulated tumors that grow slowly. As the tumor enlarges, it displaces the nerve to the periphery of the tumor, however neural function is preserved. The most common site of gastro intestinal

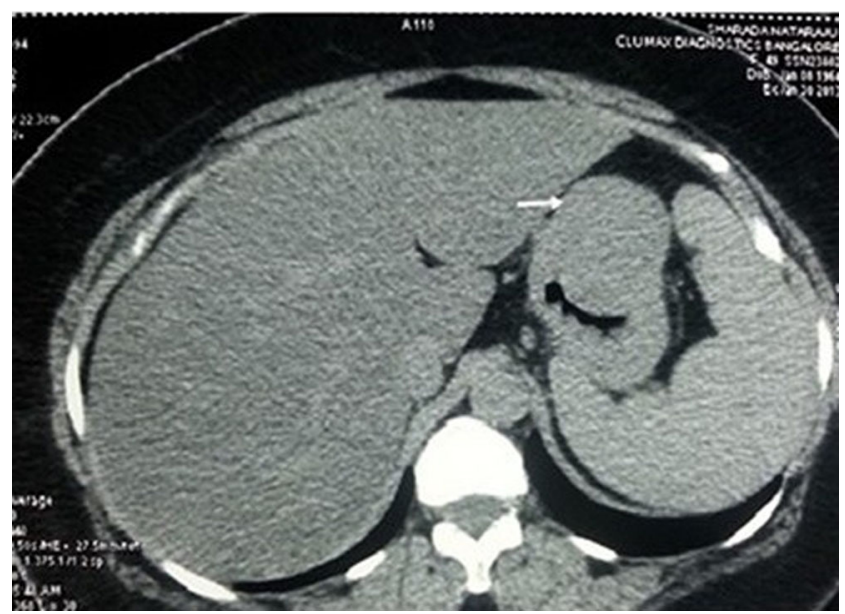

Fig. 2 CT Image of the tumor schwannoma is stomach (lesser curvature) contributing to 60 to $70 \%$ of these tumors [6]. The tumors arise most commonly from the body of the stomach (50\%), and fewer arise from the antrum (32\%) or the fundus (18\%) [7]. They arise from the nerve plexus in the submucosal plane of the stomach usually auerbach plexus or less commanly meissner plexus [8]. They occur commanly in females between third to fifth decade of life with an approximate sex ratio of 4:1 [9]. They can be asymptomatic or present as upper GI bleeding due to erosion of the overlying mucosa or even more rarely as a palpable mass. The most common presenting symptom is an episode of upper GI bleeding followed by abdominal pain [8].

Endoscopy is the investigation of choice in patients who present with upper GI bleeding. Typical finding usually includes a smooth round protruding submucosal mass with overlying ulcerated mucosa. As the lesion is submucosal endoscopic biopsies can falsely be negative.

CT scan helps in determining the extent of invasion and to differentiate a benign lesion from a malignant one [7]. On CT, schwannomas appear mostly as homogenous, strongly contrast-enhanced tumors without signs of hemorrhage, necrosis, cystic changes or calcification, in contrast to GIST [10].

The endosonographic features of schwannoma include homogeneous hypoechoic mass lesion with internal echoes with a marginal halo (without internal echogenic foci). Histopathological examination shows that the marginal halo corresponds to a lymphoid cuff [11]. In addition, the echogenicity of a schwannoma is much lower than that of the normal surrounding muscle layers. These findings may be useful for differentiating schwannoma from GIST.

Typical histologic features that assist in recognition of gastric schwannoma are focally atypical spindle cells usually arranged in a microtrabecular-microfascicular pattern, and peritumoral lymphoid cuff, often with germinal centers. Gastric schwannoma lacks encapsulation as it arises from the dispersed autonomic nerve schwann cells, as opposed to being encased by epineurium, as in soft tissue schwannoma. Vascular hyalinization with dilatation, nuclear palisading, xanthoma cells are also rare in gastric schwannoma. As gastric schwannoma are rarely malignant, pathological features like tumor size and number of mitosis per high power field have little significance. However in cases of mitosis more than 10 / $50 \mathrm{HPF}$ a watchful vigilance is advocated since the literature on malignant conversion of schwannoma is rare [12].

Both gastric schwannoma and GIST commanly present in the middle age. Both appear to have no distinct clinical features. They have the same endoscopic appearence and imaging findings. Gastric schwannoma is benign with malignant transformation being very rare where as 10 to $30 \%$ of GIST of stomach are malignant. GIST needs wide local excision with good negative surgical margin while schwannoma needs simple excision. Although definite diagnosis of gastric 
Fig. 3 Images as seen on FDG PET CT
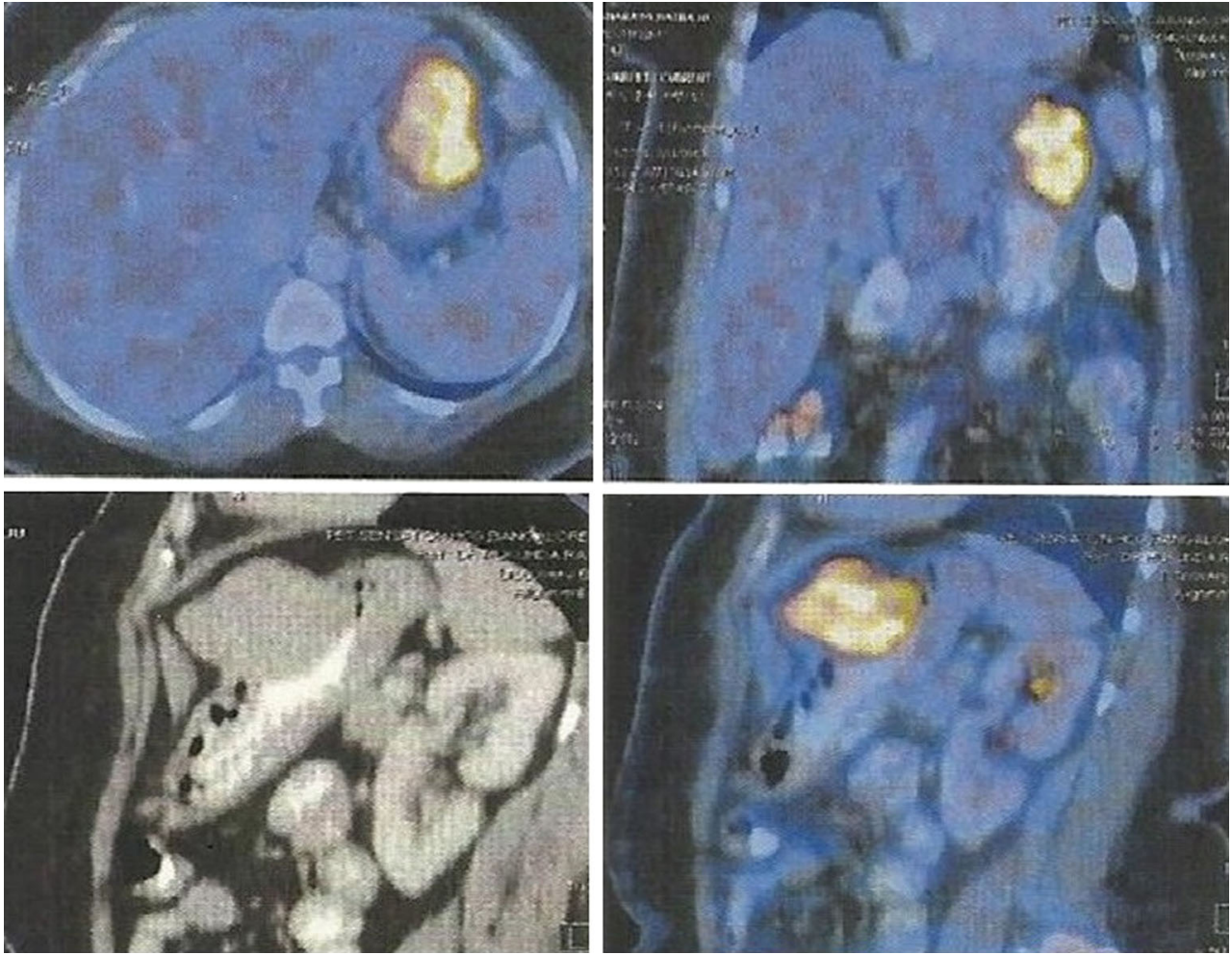

schwannoma is made by paraffin sections, frozen section shows only spindle shaped cells. Differentiation between GIST and schwannoma is often made on immunohistochemistry. Gastric schwannoma is positive for $\mathrm{S} 100$, non reactive for CD34, CD 117, Actin, HHF 35, Desmin, melan A, HMB 45 [8]. GIST shows positivity for CD 34 and CD 117 [8].

Gastrointestinal autonomic nerve tumour (GANT) is another differential diagnosis for gastric schwannoma. These are extremely rare tumors that are related to gastrointestinal autonomic nerve plexes with an aggressive course and poor outcome [13]. GANT may also be diagnosed as GIST on light microscopy (histopathology). However electron microscopy can differentiate it from GIST. They have neurosecretory granules, synapse like structure and complex long thin

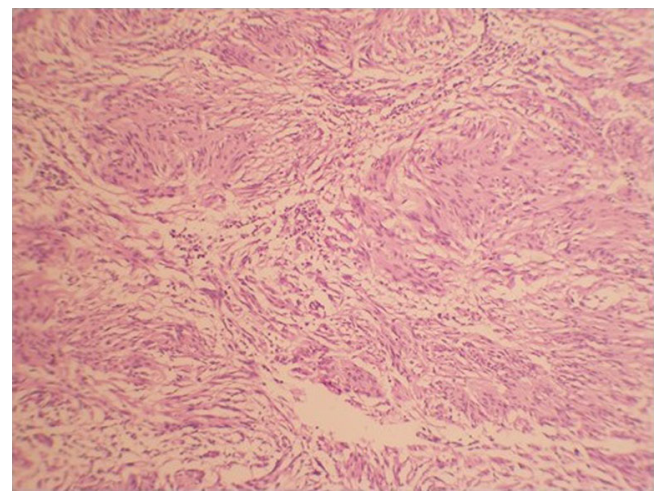

Fig. 4 Histopathological picture of the tumor showing spindle cells interdigitating cell processes joined by rudimentary cell junctions. IHC is same as GIST with $100 \%$ positivity for CD117, $58 \%$ positivity for CD34 and two out of ten positive for S100 (mean - $39 \%$ ). They are negative for actin and desmin [14]. Post operative course for benign gastric schwannoma is usually excellent with a very good prognosis.

\section{Summary}

Gastric schwanomma is a rare benign gastric neoplasm accounting to $4 \%$ of all gastric benign tumors with a female prediliction. Diagnosis is usually made incidentally. They rarely present with upper G. I. bleeding and associated abdominal pain. Preoperative differentiaition from GIST is difficult as both have same presentation. Endosonography with FNA is the investigation of choice. Simple excision is the treatment of choice. Prognosis on follow up is excellent. Malignancy is extremely rare.

\section{References}

1. Goh PMY, Lenzi JE (2001) Benign tumors of the duodenum and stomach. In: Holzheimer RG, Mannick JA (eds) Surgical treatment: evidence-based and problem-oriented. Zuckschwerdt, Munich, Available from: http://www.ncbi.nlm.nih.gov/books/NBK6948/ 
2. Miettinen M, Majidi M, Lasota J (2002) Pathology and diagnostic criteria of gastrointestinal tumors (GISTs): a review. Eur J Cancer 38: $39-51$

3. McNeer G, Pack GT, eds. (1974) Neoplasms of the stomach. Philadephia: J.B. Lippincott:518-40

4. Loffeld RJ, Balk TG, Oomen JL, van der Putten AB (1998) Upper gastrointestinal bleeding due to a malignant schwannoma of the stomach. Eur J Gastroenterol Hepatol 10:159-162

5. Gennatas CS, Exarhakos G, Kondi-Pafiti A, Kannas D, Athanassas G, Politi HD (1998) Malignant schwannoma of the stomach in a patient with neurofibromatosis. Eur J Surg Oncol 14:261-264

6. Whitehead R ed. (1989) Gastrointestinal and Oesophageal Pathology, 2 ed. New York: Churchill Livingston: 727-39

7. Burneton JN, Drouillar J, Roux P, Ettore F (1983) Neurogenic tumors of the stomach: report of 18 cases and review of the lecture. ROFOFortsch-Geb-Rontgenstr-Nuklearmed 139:192-198

8. Chen-Sung L, Han-Shui H, Chien-Ho T, Wing-Yin L, MinHsiung H (2004) Gastric schwannoma. J Chin Med Assoc 67: $583-586$
9. Sarlomo-Rikala M, Miettinen M (1995) Gastric schwannoma: a clinicopathological analysis of 6 cases. Histopathol 27:355-360

10. Levy AD, Quiles AM, Miettinen M, Sobin LH (2005) Gastrointestinal schwannomas: CT features with clinicopathologic correlation. AJR Am J Roentenol 184:797-802

11. Jung MK, Jeon SW, Cho CM, Tak WY, Kweon YO, Kim SK, Choi YH, Bae HI (2008) Gastric schwannomas: endosonographic characteristics. Abdom Imaging 33:388-390

12. VOLTAGGIO L, MURRAY R, LASOTA J, MIETTINEN M (2012) Gastric schwannoma - a clinicopathologic study of 51 cases and critical review of the literature. Hum Pathol 43(5): 650-659

13. Meshikhes AN, Al-Garni AA, Al-Momen SA, Al-Nahawi M, Subaih JA (2014) Gastrointestinal autonomic nerve tumor of the stomach. Am J Case Rep 15:7-12. doi:10.12659/AJCR.889835

14. Lee JR, Joshi V, Griffin JW Jr, Lasota J, Miettinen M (2001) Gastrointestinal autonomic nerve tumor: immunohistochemical and molecular identity with gastrointestinal stromal tumor. Am J Surg Pathol. 25(8):979-87 